\section{BSDHT AND BADT COLLABORATE IN MISSION TO PROVIDE PRESCRIPTION- ONLY MEDICINES}

UK dental hygienists and therapists have been given the green light by NHS England Chief Professionals Office to pursue its quest to be able to provide prescriptiononly medicines (POMs), specifically local anaesthetic, topical anaesthetic and fluoride varnish, to patients without first having to get a prescription from a dentist.

Over several months, the British Society of Dental Hygiene and Therapy (BSDHT) and the British Association of Dental Therapists (BADT) have collaborated to undertake a scoping process with NHS England assessing the need for use of further mechanisms to administer or supply POMs for dental hygienists and therapists.

BADT and BSDHT are pursuing exemptions to the Medicines Act.

Exemptions would mean that a list of POMs would be specified in legislation for dental therapists and dental hygienists to administer or supply.

In the future, after necessary training, this means dental hygienists and therapists would be able to provide local anaesthetic and fluoride in their daily practice without a Patient Specific Directive (PSD) or Patient Group Directive (PGD), a huge leap forward for the profession.

The BSDHT and BADT commented:

'Many of us have experienced for some time the severe limitations in our inability to supply or administer local anaesthetics and fluoride varnishes without a prescription ourselves, leading to prolonged treatment for patients and the physical and emotional impact that this has. These are not only restricting our professions' development but also our capability to provide the most timely and effective treatment for patients.

'Being given permission to finally move forward on this vital issue is fantastic news for our profession and ultimately our patients. However, this is the beginning of a long road that we need to follow with persistence and passion if we are to enforce this change.'

The BSDHT and BADT now need the considerable voice of dental hygienists and therapists to ensure they drive this vital change through and build their case of the need for the use of exemptions. They would like to hear how the inability to supply or administer certain medicines has so far impeded your ability to do your dayto-day job.

Have you had to send away a patient with incomplete treatment because you cannot get a prescription? Email prescribing@bsdht. org.uk with your experience and the impact it had on you, your patient and your practice. Speak to your colleagues, find out if this has affected them and tell BSDHT/BADT about it and help them to work hard to provide evidence for the future of their wonderful profession.

It is anticipated that the project will take at least two years and the BADT and BSDHT will be fundraising via www.justgiving.com/ crowdfunding/SubscribeToprescribe.

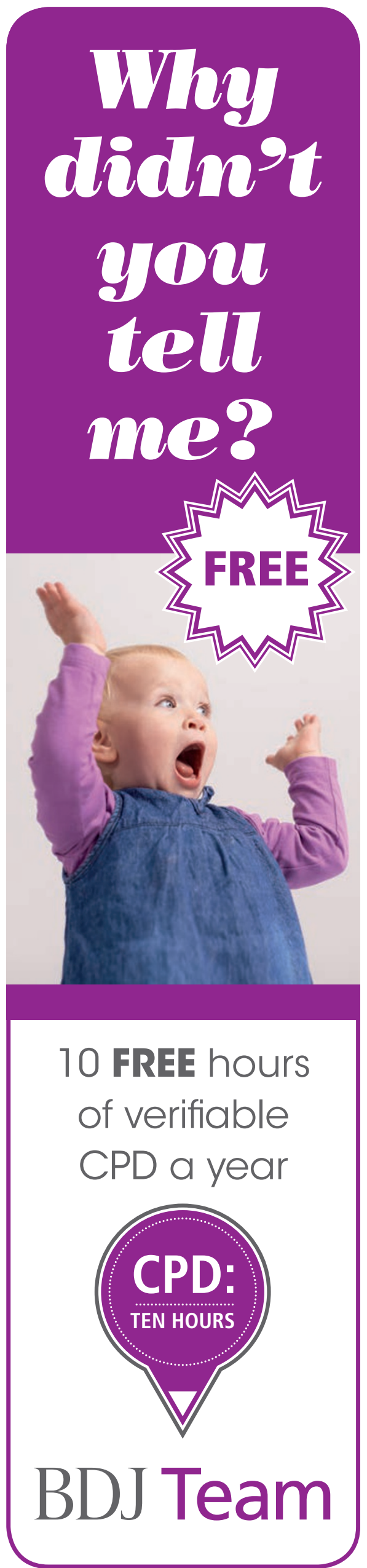

